\title{
STAMP: A Strategy-Proof Approximation Auction Mechanism for Spatially Reusable Items in Wireless Networks
}

\author{
Ruihao Zhu, Fan Wu, and Guihai Chen \\ Shanghai Key Laboratory of Scalable Computing and Systems \\ Shanghai Jiao Tong University, China \\ zhurh1992@sjtu.edu.cn; \{fwu,gchen\}@cs.sjtu.edu.cn
}

\begin{abstract}
The advent of participatory sensing markets and spectrum markets based on the wireless networks have led to a new kind of auction dealing with spatially reusable items, which can be shared by multiple parties that are geographically far apart enough from each other. Simply applying traditional auctions to spatially reusable items is vulnerable to bid manipulation, and may lead to low allocation efficiency. In this paper, we study the problem of auctioning spatially reusable items. We first propose STAMP, which is a STrategy-proof Approximation auction Mechanism for sPatially reusable items in wireless networks. STAMP can be implemented with any existing maximum independent set algorithm, and can guarantee the allocation efficiency as high as the algorithm based on. We next enhance STAMP to achieve semi-group-strategy-proofness. Finally, we implement STAMP and extensively evaluate its performance. Evaluation results show that STAMP achieves much better performance than existing mechanisms, in terms of allocation efficiency.
\end{abstract}

\section{INTRODUCTION}

Auction has been regarded as an efficient way to reallocate resources for more than two thousand years. Recently, with the rapid development of the Internet and wireless technology, many practical applications have given rise to a new trend of research, which focuses on auction mechanism design for spatially reusable items. Participatory sensing market (e.g., Sensorly and Ear-Phone) [18], [15] and spectrum auction market (e.g., FCC spectrum auctions) [12], [16] are good examples. In the participatory sensing market, the information sensed by multiple participants who are far away enough from each other is valid to the service aggregator; while in the spectrum auction market, multiple users who are not within the interference range of each other can use the same frequency band simultaneously. Unfortunately, simply applying traditional auctions to spatially reusable items is vulnerable to bid manipulation, and may lead to low allocation efficiency [22]. Therefore, it is highly needed to design novel auction mechanisms that can deal with spatially reusable items.

However, there are two major challenges when designing auction mechanisms for spatially reusable items. One challenge, which is inherited from traditional auction design, is strategy-proofness (please see Section Problem Formulation for the definition), which intuitively means that a buyer cannot get larger utility by submitting a bid other than her true valuation. A strategy-proof auction mechanism is attractive, because it can prevent the buyers from manipulating their bids, and such protects truthful buyers benefit. The other challenge is maximization of allocation efficiency (please see Section Problem Formulation for the definition). Multiple buyers who are far away enough from each other can share a spatially reusable item without splitting the value of it. A critical goal of the service aggregator who posts information sensing tasks in the participatory sensing market is to maximize the proportion of task completed in order to guarantee high quality of serve to its customers; and a goal of the auctioneer in the spectrum market is to maximize channel utilization due to the scarcity of spectrum resource. However, the optimization problem normally becomes computationally intractable when considering spatially reusable items.

In this paper, we model the problem of spatially reusable item allocation/assignment as a sealed-bid auction, and propose STAMP, which is a STrategy-proof Approximation auction Mechanism for sPatially reusable items in wireless networks. STAMP may achieve an allocation efficiency as high as the best ever known maximum independent set algorithm. Then, we enhance STAMP to resist collusion among buyers, and achieve semi-group-strategy-proofness (please see Section Semi-Group-Strategy-Proof Enhancement for the definition). The detailed contributions of this paper are as follows.

- We design a sealed-bid auction mechanism for spatially reusable items, namely STAMP, and theoretically prove that STAMP is a strategy-proof auction mechanism. STAMP can be implemented with any known maximum independent set algorithm, and therefore can achieve an allocation efficiency as high as the best ever known such algorithm.

- We further consider the problem of buyers' collusion. Since full group-strategy-proofness is shown to be impossible to achieve in online marketing scenarios unless the mechanism is posted price [8], we enhance STAMP to achieve semi-group-strategy-proofness. The enhanced STAMP maintains as good allocation efficiency as the previous version.

- We implement STAMP and compare its performance with related auction mechanisms. Our evaluation results show that STAMP achieves much higher allocation efficiency 
than existing auction mechanisms.

\section{RELATED WORKS}

To our best knowledge, there are only a few research studies on the strategy-proof auction mechanism design for participatory sensing markets. [5] designed an auction mechanism to motivate user participation. [21] designed a platform-centric model and a user-centric model for participatory sensing. Both of them did not consider the spatial reusability of the tasks. There are also a few mechanism design works for spectrum markets. [22] proposed VERITAS to ensure truthfulness in spectrum auction. However, the mechanism may suffer from low allocation efficiency due to the greedy allocation algorithm. Later, [20] proposed SMALL to achieve strategy-proofness by sacrificing a bounded number of buyers. Although SMALL shows better allocation efficiency than VERITAS, it still cannot exploit the power of the best existing maximum independent set algorithm. In contrast, our mechanism STAMP not only guarantees strategy-proofness, but also can achieve an allocation efficiency as high as the best ever known such algorithm.

There also exists a number of works on designing collusionresistant mechanisms. [17] studied the bribe-proof auction mechanism and proposed a bribe-proof auction mechanism under general case. They also proved that if the set of outcomes is finite, then a bribe-proof allocation rule should be constant. [8] gave a characterization of the impossibility result on designing full group-strategy-proof mechanism which is not posted price and presented a mechanism which retains the coalition of buyers from cheating with a high probability. They also did not consider the spatial reusability aspect. [10] investigated the buyers collusion problem in online markets and designed incentive-compatible payment mechanisms that are also resistant to collusion. [14] studied the problem of scheduling unrelated machines and presented a mechanism with verifications. However, buyers in markets of spatially reusable items are related and verification is unrealistic in auctions based on the Internet.

\section{PROBLEM FORMULATION}

We consider a spatially reusable items market (e.g., participatory sensing market and secondary spectrum market), and model it as a sealed-bid auction. There is a "seller" (e.g., service aggregator and spectrum owner), who has a spatially reusable item (e.g., information sensing task or idle channel) for sale. The item can be allocated to more than one buyer, if they are out of the conflict distance (e.g., information validation range and interference range). There is also a set of "buyers" (e.g., mobile phone users and local wireless service applications), who want to bid for the item and get profit (e.g., compensation from the service aggregator and revenue from serving her own customers). The buyers submit their sealed bids simultaneously at the beginning of the auction, such that the buyers cannot know each other's bid. We first consider a collusion free environment, and then extend our solution to resist a certain kind collusion.
In the auction, the seller has a spatially reusable item with conflict distance $d$. Denote the set of buyers by $\mathcal{N}=$ $\{1,2, \ldots, n\}$. Each buyer $i \in \mathcal{N}$ has a private valuation $v_{i}$ for the item. This is commonly known as type in the literatures. We denote the profile of buyer valuations by:

$$
\vec{v}=\left(v_{1}, v_{2}, \ldots, v_{n}\right) .
$$

Each buyer $i$ chooses her bid $b_{i}$ based on her type. Then, we denote the profile of bids by:

$$
\vec{b}=\left(b_{1}, b_{2}, \ldots, b_{n}\right) .
$$

For convenience, we let $\vec{v}_{-i}$ denote the valuation profile of buyers other than buyer $i$. Similarly, we can define $\vec{b}_{-i}$. We also use $\vec{v}_{-\mathcal{M}}\left(\vec{b}_{-\mathcal{M}}\right)$ to represent the valuation (bid) profile of buyers other than the set $\mathcal{M} \subseteq \mathcal{N}$ of buyers.

We use a graph $G=(V, E)$ to represent the conflict among buyers. Here, $V$ is the set of vertices, and $E$ is the set of edges. Each buyer is represented by a vertex in the conflict graph $G$, and there is an edge between a pair of buyers $i$ and $j$, if their geographic distance is no larger than $d$. Any pair of buyers who are connected in $G$ cannot both be the item. Here, we denote the set of neighbors of $i$ in $G$ by $N_{i}$. We can use a vector to represent the outcome of the item allocation, which is a function of $\vec{b}$ and $G$ :

$$
\begin{gathered}
\vec{x}(\vec{b}, G)=\left(x_{1}, x_{2}, \ldots, x_{n}\right), \\
\text { s.t., } x_{i}= \begin{cases}1, & \text { the item is allocated to } i ; \\
0, & \text { otherwise. }\end{cases}
\end{gathered}
$$

The auctioneer (or the seller, if the seller is trustworthy) not only determines the item allocation, but also calculates the payment profile:

$$
\vec{p}(\vec{b})=\left(p_{1}, p_{2}, \ldots, p_{n}\right) .
$$

We note that in the participatory sensing markets, the mobile phone users consume their own resource to sense information and receive compensations from the service aggregator, so the valuations (i.e., the cost induced in the process of sensing), the bids, and the payments are all negative; while in the spectrum auction markets, the buyers can get profit through providing service via the channel bought and need to pay the spectrum seller, so the valuations, the bids and the payments are positive.

We can now define the utility of buyer $i \in \mathcal{N}$ as the difference between her valuation and payment:

$$
u_{i}(\vec{b})=x_{i} v_{i}-p_{i}(\vec{b}) \text {. }
$$

We consider that the buyers are rational and each buyer's goal is to maximize her own utility.

We now recall the definition of Dominant Strategy [13], [7], Strategy-Proofness [11] and Allocation Efficiency, which will be used in the following parts of this paper.

Definition 1 (Dominant Strategy). Strategy $a_{i}$ is a player $i$ 's dominant strategy, if for any $a_{i}^{\prime} \neq a_{i}$ and any strategy profile of the other players $a_{-i}$,

$$
u_{i}\left(a_{i}, a_{-i}\right) \geq u_{i}\left(a_{i}^{\prime}, a_{-i}\right)
$$


The concept of incentive-compatibility is based on dominant strategy. It means that revealing truthful information is the dominant strategy for every player. A company concept is individual-rationality, which intuitively means that for every player who truthfully participates the auction is expected to gain non-negative utility. We now can introduce the definition of Strategy-Proof Mechanism.

Definition 2 (Strategy-Proof Mechanism). A mechanism is strategy-proof when it satisfies both incentive-compatibility and individual-rationality.

Definition 3 (Allocation Efficiency). The allocation efficiency of an auction mechanism is the total number of items the buyers win.

The design goal of our auction mechanism is to achieve strategy-proofness and allocation efficiency maximization. Although the problem of allocation efficiency maximization can be formulated as the following binary program:

Objective:

$$
\text { Maximize } \sum_{k=1}^{n} x_{k}
$$

Subject to:

$$
\begin{array}{lr}
x_{i}+x_{j} \leq 1, & \forall(i, j) \in E ; \\
x_{i} \in\{0,1\}, & \forall i \in \mathcal{N},
\end{array}
$$

it is known that the problem is computationally intractable [19]. Therefore, we should seek for an approximation approach.

\section{DESIGN OF STAMP}

In this section, we present the design of STAMP, and prove its strategy-proofness and approximation ratio of allocation efficiency. STAMP consists of an item allocation algorithm and a payment scheme. The item allocation algorithm comprises initial item allocation and item reallocation algorithms, STAMP can achieve an allocation efficiency as good as the best known maximum independent set algorithm by allowing to use any such algorithm. By performing the item reallocation algorithm, the mechanism allocates items to buyers with higher valuations. In the payment scheme, by charging the buyers the minimum value by bidding which she can still win the item, STAMP guarantees strategy-proofness.

\section{A. Item Allocation}

STAMP starts with the initial item allocation algorithm. During the initial item allocation, STAMP allocates the item using the best known maximum independent set approximation algorithm for general bounded degree graphs [6], [3], so that the buyers who are connected in $G$ are not allocated the item concurrently, i.e.:

$$
x_{i}+x_{j} \leq 1, \forall(i, j) \in E .
$$

STAMP then performs item reallocation algorithm. It visits all the buyers iteratively, from the smallest index to the highest index (i.e., from 1 to $n$ ).
Assume that STAMP is attempting to perform the item reallocation for $i$ (if $i$ is allocated an item). STAMP finds a set of buyers $w_{i}$ such that:

$$
\begin{aligned}
& \forall l \in w_{i}: \\
& \text { 1. } l \in N_{i} . \\
& \text { 2. } x_{l}=0 . \\
& \text { 3. } x_{k}=0, \forall k \in N_{l} . \\
& \text { 4. } l \notin w_{j}, \forall j \in\{1,2, \ldots, i-1\} . \\
& \text { 5. } l \geq i .
\end{aligned}
$$

Here, constraint (2) guarantees that the buyers who are connected are not allocated the item concurrently. Constraint (3) and (4) jointly guarantee that the buyers selected in $w_{i}$ are all able to be allocated the item. Constraint (5) and (6) jointly guarantee that STAMP's allocation is monotone [1].

If $w_{i} \neq \varnothing$, for the buyers who bid higher than $i$ in $w_{i}$, STAMP allocates the item to them instead of $i$. The allocation outcome of the other buyers remains the same. STAMP then visit the next buyer, i.e.:

$$
x_{i}=0, x_{j}=1, \forall j \in w_{i}, b_{j}>b_{i} .
$$

If $w_{i}=\varnothing$, STAMP visits the next buyer.

The item reallocation algorithm runs until all the buyers are visited exactly once.

The algorithm for item allocation is formally stated in Algorithm 1. Function $M I S(G)$ can be any existing maximum independent set algorithm and it allocates the item to the corresponding buyers. Algorithm 1 returns the allocation result and $\vec{w}$, which is used in the payment determination.

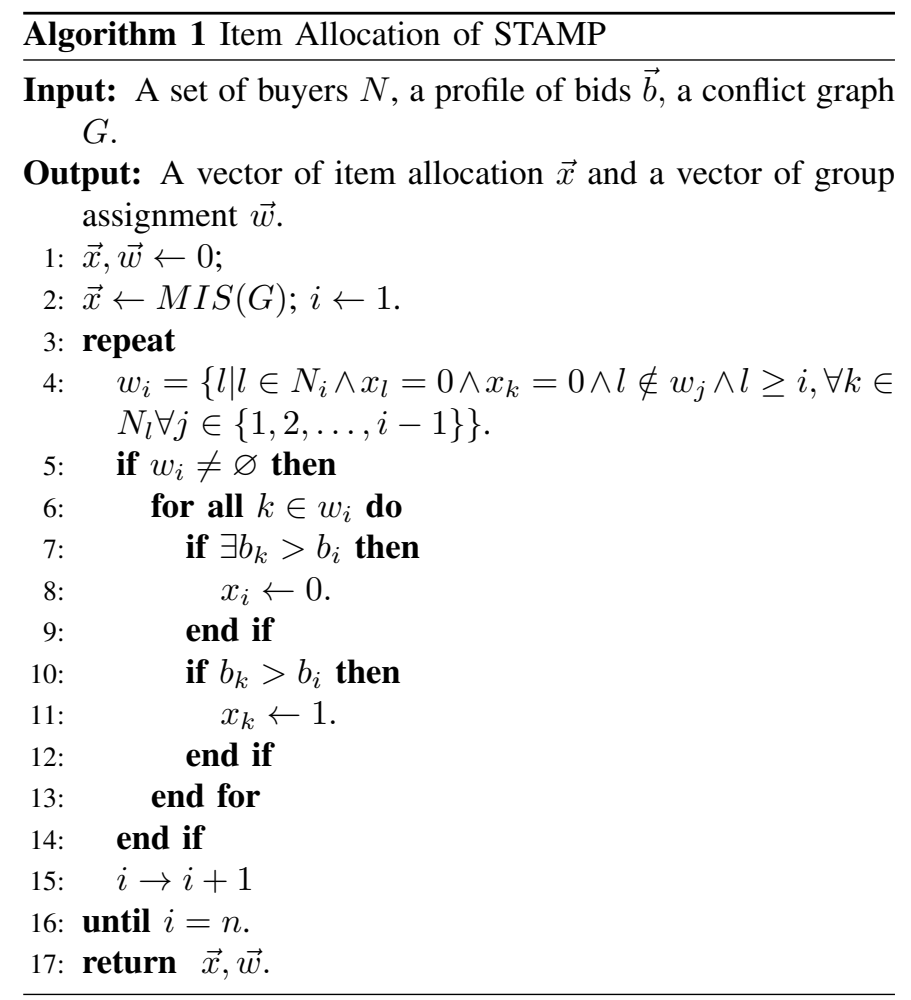


The computational complexity of the maximum independent set algorithm is $O\left(n^{\kappa}\right)$ [6]. Notice that there is only one round of reallocation, so there are at most $n$ times of reallocations. The computational complexity for reallocation is $O\left(n^{2}\right)$. The mechanism takes a $O\left(n^{2+\kappa}\right)$ time to determine which buyers are allocated the item.

\section{B. Payment Determination}

In this subsection, we present the payment scheme of STAMP. For a winning buyer, STAMP charges her the minimum price that she can still win by bidding this value (this is commonly known as the critical value [22]) and the buyers who are not allocated the price 0 :

$$
p_{i}(\vec{b})= \begin{cases}\underset{b_{i}}{\operatorname{argmin}}\left(x_{i}=1\right), & \text { the item is allocated to } i \\ 0, & \text { otherwise. }\end{cases}
$$

Combining the result of $\vec{w}$ of Algorithm 1, the value of argmin $\left(x_{i}=1\right)$ is computed as following:

$$
\begin{aligned}
& \underset{b_{i}}{\operatorname{argmin}}\left(x_{i}=1\right)=\max (A, B), \\
& \text { Where } A= \begin{cases}b_{j}, & \text { if } i \in w_{j} \text { for some } j \\
0, & \text { otherwise. }\end{cases} \\
& B=\max _{k \in w_{i}} b_{k} \text {. }
\end{aligned}
$$

We can now draw the following lemmas and theorems:

\section{Lemma 1. STAMP's allocation algorithm satisfies monotonic-} ity.

Proof: For an arbitrary buyer $i$, we distinguish two cases:

- If $i$ is allocated in the initial item allocation, she is not contained in any $w_{j}$, then if she bids higher than all the buyers in $w_{i}$, she wins the item; otherwise, she does not win the item.

- If $i$ is not allocated in the initial item allocation, she is contained in exactly one $w_{j}$. In order to win the item, she first needs to bid higher than buyer $j$. What's more, she also needs to bid higher than all the buyers in $w_{i}$, or she still does not win the item. Therefore, if she bids higher than the larger one of $b_{j}$ and the highest bid in $w_{i}$, she wins the item; otherwise, she does not win the item.

According to the above analysis, we can draw the conclusion that the allocation algorithm of STAMP is monotone.

The monotonicity of STAMP's allocation algorithm and the critical payment scheme guarantee STAMP's incentive compatibility [1].

\section{Lemma 2. STAMP achieves incentive-compatibility.}

Proof: For a buyer $i$, we prove that she cannot increases her utility by misreporting. We divide the proof into two cases:

1) If $i$ 's valuation satisfies $v_{i} \geq \underset{b_{i}}{\operatorname{argmin}}\left(x_{i}^{f}=1\right)$, then $i$ is allocated the item when bidding truthfully. We can further distinguish two cases of misreporting:
- $i$ misreports $b_{i}^{\prime}\left(>v_{i}\right)$, then $i$ is still allocated the item, her utility remains unchange:

$$
\begin{aligned}
& u_{i}\left(b_{i}^{\prime}, \vec{b}_{-i}\right) \\
= & v_{i} x_{i}-\underset{b_{i}}{\operatorname{argmin}}\left(x_{i}=1\right) \\
= & u_{i}(\vec{b}) .
\end{aligned}
$$

- $i$ bids $b_{i}^{\prime}\left(<v_{i}\right)$, then her utility remains unchange or becomes 0 :

$$
u_{i}\left(b_{i}^{\prime}, \vec{b}_{-i}\right)= \begin{cases}v_{i} x_{i}-\underset{b_{i}}{\operatorname{argmin}}\left(x_{i}=1\right), & i \text { wins; } \\ 0, & \text { otherwise. }\end{cases}
$$

2) If $i$ 's valuation for winning the item is $v_{i}<$ $\operatorname{argmin}\left(x_{i}=1\right)$, then $i$ is not allocated the item when $b_{i}$

bidding truthfully. We can further distinguish two cases of misreporting:

- $i$ misreports $b_{i}^{\prime}\left(<v_{i}\right)$, then $i$ is still not allocated the item, her utility remains unchange:

$$
u_{i}\left(b_{i}^{\prime}, \vec{b}_{-i}\right)=0=u_{i}(\vec{b}) .
$$

- $i$ bids $b_{i}^{\prime}\left(>v_{i}\right)$, then her utility remains unchange or even negative:

$$
u_{i}\left(b_{i}^{\prime}, \vec{b}_{-i}\right)= \begin{cases}v_{i} x_{i}-\underset{b_{i}}{\operatorname{argmin}}\left(x_{i}=1\right), & i \text { wins; } \\ 0, & \text { otherwise. }\end{cases}
$$

Thus we can conclude that the buyers' dominant strategy is bidding truthfully and STAMP achieves incentive compatibility.

Lemma 3. STAMP achieves individual-rationality.

Proof: For a buyer $i$, assume that she bids truthfully by $b_{i}=v_{i}$. We divide the proof into two cases:

1) If $b_{i}=v_{i} \geq \operatorname{argmin}\left(x_{i}=1\right)$, then $i$ is allocated the item and her utility is non-negative:

$$
\begin{aligned}
u_{i}(\vec{b}) & =v_{i} x_{i}-p_{i}(\vec{b}) \\
& =v_{i}-\underset{b_{i}}{\operatorname{argmin}}\left(x_{i}=1\right) \\
& \geq 0 .
\end{aligned}
$$

2) If $b_{i}=v_{i}<\underset{b_{i}}{\operatorname{argmin}}\left(x_{i}=1\right)$, then $i$ is not allocated the item and her utility is zero:

$$
u_{i}(\vec{b})=0 .
$$

Since the utility of the buyers are non-negative when they bids truthfully, we can conclude that STAMP achieves individual rationality.

Theorem 1. STAMP achieves strategy-proofness.

Proof: This is clear from Lemma 2, Lemma 3 and the definition of strategy-proof auction mechanism.

Theorem 2. STAMP achieves an asymptotic approximation ratio of:

$$
\min \left\{\kappa / \mu,\left[\kappa^{\prime} \log (\log \Delta)\right] / \Delta\right\}
$$


(where $\kappa$ is a positive constant, $\kappa^{\prime}$ is a constant depends on $\kappa$, and $\Delta, \mu$ are the maximum and the average degrees of the $G$, respectively) in terms of allocation efficiency [6].

Proof: During the item reallocation procedure, there is no decrease in the allocation efficiency, so the approximation ratio of the allocation efficiency is solely determined by the initial item allocation algorithm. Since the initial item allocation is equivalent to the maximum independent set algorithm in $G$ and the asymptotic approximation ratio of the best ever known algorithm [6] is:

$$
\min \left\{\kappa / \mu,\left[\kappa^{\prime} \log (\log \Delta)\right] / \Delta\right\}
$$

(where $\kappa$ is a positive constant, $\kappa^{\prime}$ is a constant depends on $\kappa$, and $\Delta, \mu$ are the maximum and the average degrees of the $G$, respectively), we can conclude this statement.

C. A Toy Example

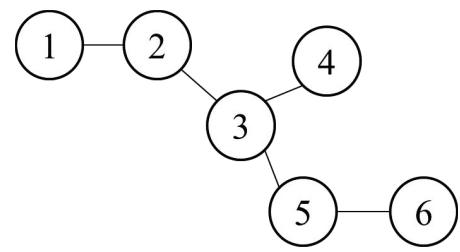

Fig. 1. A Toy Example

We now give a toy example to illustrate how STAMP works. Suppose that we have a set of $n=6$ buyers and the profile of the valuations and bids is:

$$
\vec{v}=\vec{b}=(3,1,4,5,3,1) .
$$

The graph $G$ is shown in Figure 1. Suppose that after the initial item allocation, buyer 1,3 and 6 is allocated the item:

$$
\vec{x}=(1,0,1,0,0,1) \text {. }
$$

Then STAMP performs the item reallocation:

1) For buyer $1, w_{1}=\{2\}$ and $b_{2} \leq b_{1}$, so she wins the item with payment 1 .

2) For buyer 2, she is not allocated the item so her payment is 0 .

3) For buyer $3, w_{3}=\{4,5\}$. The only reallocation is between buyer 3 and buyer 4 :

$$
\vec{x}=(1,0,0,1,0,1) \text {. }
$$

4) For buyer $4, w_{4}=\varnothing$. She wins the item with payment 4.

5) For buyer 5 , she is not allocated the item so her payment is 0 .

6) For buyer $6, w_{6}=\varnothing$. She wins the item with payment 0 .

The allocation and payment vectors are:

$$
\vec{x}=(1,0,0,1,0,1), \vec{p}=(1,0,0,4,0,0) .
$$

\section{Semi-Group-Strategy-Proof Enhancement}

One important threat of auction markets is that buyers may form coalitions and gain extra profit through strategically manipulating their bids. Due to the fact that buyers collusion is hard to detect and it can severely hurt the truthfully bidding buyers' and the seller's incentives, designing group-strategyproof auction mechanisms is of great importance. However, it is shown that full group-strategy-proofness is impossible in online marketing scenarios unless the mechanism is posted price [8] and such mechanism may significantly lower the allocation efficiency. Therefore, we enhance STAMP to achieve semi-group-strategy-proofness, in which any buyer and any coalition of buyers cannot gain extra profit by bidding lower than their true valuations. The enhanced STAMP can achieve as good allocation efficiency as the original one.

For simplicity, we only consider the case that all the valuations, bids and payments are positive (for the case of negative valuations, bids and payments, the mechanism can be derived similarly). We assume that every buyer's valuation and bid is bounded:

$$
v_{\text {inf }} \leq v_{i}, b_{i} \leq v_{\text {sup }}, \forall i \in \mathcal{N}
$$

In order to achieve semi-group-strategy-proofness, we define a monotone nondecreasing function [14], which is a function $m()$ that associates to the allocation outcome and the buyers' bids. If one buyer increases her bid and all the others remain the same, the function does not decrease:

$$
\begin{aligned}
m\left(\vec{x}\left(\left(b_{i}^{\prime}, \vec{b}_{-i}\right), G\right),\left(b_{i}^{\prime}, \vec{b}_{-i}\right)\right) & \geq m(\vec{x}(\vec{b}, G), \vec{b}) \\
& \forall b_{i}^{\prime}\left(>b_{i}\right), \vec{b}_{-i} .
\end{aligned}
$$

We give the formal definition of semi-group-strategy-proof mechanism [9] :

Definition 4 (Semi-Group-Strategy-Proof Mechanism). We say a mechanism $(\vec{x}(\vec{b}, G), \vec{p}(\vec{b}))$ is semi-group-strategy-proof if every strategy for a single buyer or any coalition of buyers that involves understating the value is dominated by a strategy that does not understate the value and it achieves individual rationality.

We enhance STAMP so that it can effectively solve the problem. Enhanced STAMP still consists of an item allocation algorithm and a payment scheme. The item allocation algorith$\mathrm{m}$ comprises initial allocation algorithm and item reallocation algorithm. Enhanced STAMP adopts the initial algorithm of STAMP and design a new item reallocation algorithm together with a new payment scheme which associates the payment of a buyer with the global bids to achieve semi-group-strategyproofness while preserving the same allocation efficiency as STAMP.

\section{A. Enhanced Item Allocation}

Enhanced STAMP starts with the initial item allocation algorithm. It allocates the item using the best known maximum independent set algorithm so that the buyers who are connected in $G$ are not allocated the item concurrently. 
Next, enhanced STAMP performs the item reallocation. Denote the buyers allocated in the initial allocation by:

$$
\mathcal{Q}=\left\{i_{1}, i_{2} \ldots, i_{q}\right\}
$$

Where $q$ is the number of allocated buyers. Starting from $i_{1}$ to $i_{q}$, enhanced STAMP finds a path for each of them. For buyer $i_{l} \in \mathcal{Q}$, enhanced STAMP starts from it and adds buyers to form a path (breaking ties randomly) in $G$ iteratively so that no buyer added to this path belongs to another selected path, nor does any of them is connected with the buyers in another selected path. This procedure runs until no new buyer can be added. We denote the path by $\sigma_{i_{l}}$ :

$$
\begin{aligned}
& \forall j \in \sigma_{i_{l}}: \\
& 1 . j \notin \sigma_{k}, \forall k \neq i_{l} . \\
& 2 . j^{\prime} \notin \sigma_{k}, \forall j^{\prime} \in N_{j} \forall k \neq i_{l} .
\end{aligned}
$$

Denote the set of paths by:

$$
\sigma=\left\{\sigma_{i_{1}}, \sigma_{i_{2}}, \ldots, \sigma_{i_{q}}\right\}
$$

Enhanced STAMP then reallocates the item iteratively. For $\sigma_{i_{j}}$, enhanced STAMP reallocates the item to the one who bids the highest of this path and breaking ties randomly. Enhanced STAMP performs the reallocation algorithm until all the paths in $\sigma$ are visited exactly once.

\section{B. Enhanced Payment Determination}

Denote the set of winning buyers by $\mathcal{W}$ :

$$
\mathcal{W}=\left\{i \mid i \in \mathcal{N}, x_{i}=1\right\} .
$$

Enhanced STAMP first defines the monotone nondecreasing function (see Lemma 3 for the proof of the monotone nondecreasing of this function):

$$
m(\vec{x}(\vec{b}, G), \vec{b})=\frac{\sum_{k \in \mathcal{W}} b_{k}}{\hat{k}},
$$

where $\hat{k}$ is a bounded constant:

$$
\hat{k} \geq \frac{\left(v_{\text {sup }}-v_{\text {inf }}\right)}{v_{\text {inf }}} \times q .
$$

Since the bids are bounded, so does the function $m(\vec{x}(\vec{b}, G), \vec{b})$. Assume that the lower bound and upper bound are $\beta_{\text {inf }}$ and $\beta_{\text {sup }}$, respectively:

$$
\frac{q \times v_{\text {inf }}}{\hat{k}} \leq \beta_{\text {inf }} \leq m(\vec{x}(\vec{b}, G), \vec{b}) \leq \beta_{\text {sup }} \leq \frac{q \times v_{\text {sup }}}{\hat{k}} .
$$

Enhanced STAMP associates the payment of a winning buyer to the bids of the other buyers and charges the buyers who are not allocated 0:

$p_{i}(\vec{b})= \begin{cases}\hat{h}-\frac{\sum_{j \in(\mathcal{W} \backslash\{i\})} b_{j}}{\hat{k}} & \text { the item is allocated to } i \\ 0, & \text { otherwise. }\end{cases}$

Where $\hat{h}$ is a constant and satisfies that: 1. Every buyer's payment is positive; 2. No buyer is charged higher than her valuation:

$$
\beta_{\text {sup }}-\frac{v_{\text {inf }}}{\hat{k}} \leq \hat{h} \leq \beta_{\text {inf }}+\frac{(k-1) v_{\text {inf }}}{\hat{k}} .
$$

By eqn. 10 and 11, inequality 12 is validate.

We can draw the following lemmas and theorems:

Lemma 4. The function $m()$ defined in eqn. 9 is actually monotone nondecreasing.

Proof: For buyer $i$ and her bid $b_{i}$. We divide the proof into three cases:

1) If she is allocated the item by bidding $b_{i}$, and misreports her bid $b_{i}^{\prime}\left(>b_{i}\right)$. According to eqn. 7 and eqn. 8, $i$ does not change the item allocation outcome and the function increases or remains the same:

$$
\begin{aligned}
& m\left(\vec{x}\left(\left(b_{i}^{\prime}, \vec{b}_{-i}\right), G\right),\left(b_{i}^{\prime}, \vec{b}_{-i}\right)\right) \\
= & \frac{\sum_{j \neq i, j \in \mathcal{W}} b_{j}+b_{i}^{\prime}}{\hat{k}} \\
\geq & \frac{\sum_{j \in \mathcal{W}} b_{j}}{\hat{k}} \\
= & m(\vec{x}(\vec{b}, G), \vec{b}) .
\end{aligned}
$$

2) If she is not in any path in $\sigma$, she is not allocated and not able to affect the allocation outcome. The function remains the same.

3) If she is not allocated the item by bidding $b_{i}$, but she is in a $\sigma_{k}$. we can further distinguish two cases:

- If she bids $b_{i}^{\prime}\left(>b_{i}\right)$, but $b_{i}^{\prime}<\max \left\{b_{j} \mid j \in \sigma_{k}\right\}$, then she still does not win the item and the function remains the same:

$$
\begin{aligned}
& m\left(\vec{x}\left(\left(b_{i}^{\prime}, \vec{b}_{-i}\right), G\right),\left(b_{i}^{\prime}, \vec{b}_{-i}\right)\right) \\
= & \frac{\sum_{j \in \mathcal{W}} b_{j}}{\hat{k}} \\
= & m(\vec{x}(\vec{b}, G), \vec{b}) .
\end{aligned}
$$

- If she bids $b_{i}^{\prime}\left(\geq \max \left\{b_{j} \mid j \in \sigma_{k}\right\}\right)$, then according to the reallocation rule, she is allocated the item and $i$ 's new bid does not affect the allocation outcome for all the buyers except for herself and the one who originally bids the highest in $\sigma_{k}$. The function increases or remains the same:

$$
\begin{aligned}
& m\left(\vec{x}\left(\left(b_{j}^{\prime}, \vec{b}_{-i}\right), G\right),\left(b_{i}^{\prime}, \vec{b}_{-i}\right)\right) \\
= & \frac{\sum_{j \neq i, j \in \mathcal{W}^{\prime}} b_{j}+b_{i}^{\prime}}{\hat{k}} \\
\geq & \frac{\sum_{j \neq i, j \in \mathcal{W}^{\prime}} b_{j}+\max _{j \in \sigma_{k} \backslash\{i\}} b_{j}}{\hat{k}} \\
= & \frac{\sum_{j \in \mathcal{W}} b_{j}}{\hat{k}} \\
= & m(\vec{x}(\vec{b}, G), \vec{b}) .
\end{aligned}
$$

Where $\mathcal{W}^{\prime}$ is the new set of winning buyers:

$$
\left.\mathcal{W}^{\prime}=(\mathcal{W} \bigcup\{i\}) \backslash \underset{j \in\left(\sigma_{k} \backslash\{i\}\right)}{\operatorname{argmax}}\left(b_{j}\right)\right\} .
$$

From the above analysis, we finish the proof. 
Lemma 5. For a buyer $i$ and every $\vec{b}$ and $\vec{b}^{\prime}=\left(v_{i}, \vec{b}_{-i}\right)$ such that $b_{i} \leq v_{i}$ and $\vec{b}_{-i}=\vec{b}_{-i}^{\prime}$. We have:

$$
u_{i}(\vec{b}) \leq u_{i}\left(\vec{b}^{\prime}\right)
$$

Proof: According to Lemma 3, we distinguish two cases:

1) $i$ is allocated when bidding $b_{i}$ and she does not affect the allocation outcome by bidding $b_{i}^{\prime}=v_{i}\left(\geq b_{i}\right)$. Her utility remains the same:

$$
\begin{aligned}
& u_{i}\left(\vec{b}^{\prime}\right) \\
= & v_{i} x_{i}-p_{i}\left(\vec{b}^{\prime}\right) \\
= & v_{i} x_{i}-p_{i}(\vec{b}) \\
= & u_{i}(\vec{b}) .
\end{aligned}
$$

2) $i$ is not allocated when bidding $b_{i}$. We further distinguish two cases:

- $i$ does not win by bidding $v_{i}$. Her utility remains 0 :

$$
u_{i}\left(\vec{b}^{\prime}\right)=0=u_{i}(\vec{b})
$$

- $i$ is allocated by bidding $b_{i}^{\prime}=v_{i}$. By eqn. 12 , we have:

$$
\begin{aligned}
& u_{i}\left(\vec{b}^{\prime}\right) \\
= & v_{i} x_{i}-p_{i}\left(\vec{b}^{\prime}\right) \\
= & m\left(\vec{x}\left(\vec{b}^{\prime}, G\right), \vec{b}^{\prime}\right)+\frac{k-1}{k} v_{i}-\hat{h} \\
\geq & \beta_{\text {inf }}+\frac{k-1}{k} v_{\mathrm{inf}}-\hat{h} \\
\geq & 0 \\
= & u_{i}(\vec{b}) .
\end{aligned}
$$

By the above analysis, we finish the proof.

Lemma 6. For a coalition of buyers $C$ of any size and every $\vec{b}$ and $\vec{b}^{\prime}$ such that $b_{i} \leq b_{i}^{\prime} \leq v_{i}, \forall i \in C$ and $b_{j}=b_{j}^{\prime}, \forall j \in \mathcal{N} \backslash C$. We have:

$$
\sum_{i \in C} u_{i}(\vec{b}) \leq \sum_{i \in C} u_{i}\left(\vec{b}^{\prime}\right)
$$

Proof: For a buyer $j \in C$ We distinguish three cases:

1) If $j$ is not in any element of $\sigma_{k}$, then her bid does not affect the allocation outcome and the sum of utilities of the coalition remains the same.

2) If $j$ is allocated the item by bidding $b_{j}$, then she does not change the allocation result by bidding $b_{j}^{\prime}=v_{j}\left(\geq b_{j}\right)$. The sum of utilities of the coalition increases or remains the same:

$$
\begin{aligned}
& \sum_{i \in C} u_{i}(\vec{b}) \\
& =\sum_{i \in C}\left(v_{i}-p_{i}(\vec{b})\right) \\
& =\sum_{i \in C \cap \mathcal{W}}\left(v_{i}-\hat{h}+\frac{\sum_{l \in(\mathcal{W} \backslash\{i\})} b_{l}}{\hat{k}}\right) \\
& =\sum_{i \in C \cap \mathcal{W}} v_{i}+|C \bigcap \mathcal{W}-1| \cdot(m(\vec{x}(\vec{b}, G), \vec{b})-\hat{h}) \\
& -\frac{1}{k} \cdot\left(\sum_{i \in(\mathcal{W} \cap C \backslash\{j\})} b_{i}\right)-\hat{h}+\frac{\sum_{l \in(\mathcal{W} \backslash\{j\})} b_{l}}{\hat{k}} \\
& \leq|C \bigcap \mathcal{W}-1| \cdot\left(m\left(\vec{x}\left(\left(v_{j}, \vec{b}_{-j}\right), G\right),\left(v_{j}, \vec{b}_{-j}\right)\right)-\hat{h}\right) \\
& +\sum_{i \in C \cap \mathcal{W}} v_{i}-\frac{1}{k} \cdot\left(\sum_{i \in(\mathcal{W} \cap C) \backslash\{j\}} b_{i}\right)-\hat{h} \\
& +\frac{\sum_{l \in(\mathcal{W} \backslash\{j\})} b_{l}}{\hat{k}} \\
& =\sum_{i \in C \cap \mathcal{W} \backslash\{j\}}\left(v_{i}-\hat{h}+\frac{\sum_{l \in(\mathcal{W} \backslash\{i, j\})} b_{l}+v_{j}}{\hat{k}}\right) \\
& +\left(v_{j}-\hat{h}+\frac{\sum_{l \in(\mathcal{W} \backslash\{j\})} b_{l}}{\hat{k}}\right) \\
& =\sum_{i \in C \backslash\{j\}}\left(v_{i}-p_{i}\left(\left(v_{j}, \vec{b}_{-j}\right)\right)+\left(v_{j}-p_{j}\left(v_{j}, \vec{b}_{-j}\right)\right)\right. \\
& =\sum_{i \in C} u_{i}\left(v_{j}, \vec{b}_{-j}\right) .
\end{aligned}
$$

3) If $j$ is not allocated the item and she is in $\sigma_{k}$, we denote that:

$$
a=\underset{l \in \sigma_{k}}{\operatorname{argmax}}\left(b_{l}\right) .
$$

We can further distinguish two cases (here we assume that $a \in C$ and for the case $a \notin C$, the proof is similar):

- If $b_{a} \geq b_{j}^{\prime}=v_{j} \geq b_{j}, \mathrm{j}$ does not affect the allocation outcome and the sum of utilities of the coalition remains the same:

$$
\sum_{i \in C} u_{i}(\vec{b})=\sum_{i \in C} u_{i}\left(\left(v_{j}, \vec{b}_{-j}\right)\right)
$$

- If $b_{j}^{\prime}=v_{j} \geq b_{a} \geq b_{j}$, then $\mathbf{j}$ gets the item by bidding $b_{j}^{\prime}=v_{j}$. The sum of utilities of the coalition increases or remains the same if both $j$ and $a$ bid their true values (due to the item allocation rule of mechanism ${ }^{\text {semi }}$, they do not affect the allocation 
result of other buyers):

$$
\begin{aligned}
& \sum_{i \in C} u_{i}(\vec{b}) \\
& =\sum_{i \in C}\left(v_{i}-p_{i}(\vec{b})\right) \\
& =\sum_{i \in C \cap \mathcal{W}}\left(v_{i}-\hat{h}+\frac{\sum_{l \in(\mathcal{W} \backslash\{i\})} b_{l}}{\hat{k}}\right) \\
& =\sum_{i \in C \cap \mathcal{W}} v_{i}+|C \bigcap \mathcal{W}| \cdot(m(\vec{x}(\vec{b}, G), \vec{b})-\hat{h}) \\
& -\frac{1}{k} \cdot\left(\sum_{i \in(\mathcal{W} \cap C)} b_{i}\right)-\hat{h} \\
& =|C \bigcap \mathcal{W}-1| \cdot(m(\vec{x}(\vec{b}, G), \vec{b})-\hat{h}) \\
& +\sum_{i \in(C \cap \mathcal{W}) \backslash\{a\}} v_{i}-\frac{1}{k} \cdot\left(\sum_{i \in(C \cap \mathcal{W}) \backslash\{a\}} b_{i}\right) \\
& +v_{a}-\hat{h}+\frac{\sum_{i \in(\mathcal{W} \backslash\{a\})} b_{i}}{\hat{k}} \\
& \leq|C \bigcap \mathcal{W}-1| \cdot\left(m \left(\vec{x}\left(\left(v_{j}, v_{a}, \vec{b}_{-\{j, a\}}\right), G\right),\right.\right. \\
& \left.\left.\left(v_{j}, v_{a}, \vec{b}_{-\{j, a\}}\right)\right)-\hat{h}\right) \\
& +\sum_{i \in(C \cap \mathcal{W}) \backslash\{a\}} v_{i}-\frac{1}{k} \cdot\left(\sum_{i \in C \cap \mathcal{W} \backslash\{a\}} b_{i}\right) \\
& +\max \left(v_{j}, v_{a}\right)-\hat{h}+\frac{\sum_{i \in(\mathcal{W} \backslash\{j, a\})} b_{i}}{\hat{k}} \\
& =\sum_{i \in C \cap \mathcal{W} \backslash\{j, a\}}\left(v_{i}-\hat{h}+\frac{\sum_{l \in(\mathcal{W} \backslash\{i, j, a\})} b_{l}}{\hat{k}}\right. \\
& \left.+\frac{\max \left(v_{j}, v_{a}\right)}{\hat{k}}\right)+\max \left(v_{j}, v_{a}\right)-\hat{h} \\
& +\frac{\sum_{i \in(\mathcal{W} \backslash\{j, a\})} b_{i}}{\hat{k}} \\
& =\sum_{i \in C \cap \mathcal{W} \backslash\{j, a\}}\left(v_{i}-p_{i}\left(\left(v_{j}, v_{a}, \vec{b}_{-\{j, a\}}\right)\right)\right) \\
& +\max \left(v_{j}-p_{j}\left(\left(v_{j}, v_{a}, \vec{b}_{-\{j, a\}}\right)\right),\right. \\
& \left.v_{a}-p_{a}\left(\left(v_{j}, v_{a}, \vec{b}_{-\{j, a\}}\right)\right)\right) \\
& =\sum_{i \in C} u_{i}\left(\left(v_{j}, v_{a}, \vec{b}_{-(j, a)}\right)\right) .
\end{aligned}
$$

In all above three cases, we can conclude that:

$$
\sum_{i \in C} u_{i}(\vec{b}) \leq \sum_{i \in C} u_{i}\left(\left(v_{j}, \vec{b}_{-j}\right)\right), \forall \vec{b}_{-j} .
$$

or

$$
\sum_{i \in C} u_{i}(\vec{b}) \leq \sum_{i \in C} u_{i}\left(\left(v_{j}, v_{a}, \vec{b}_{-\{j, a\}}\right)\right), \forall \vec{b}_{-\{j, a\}} .
$$

Thus we fix $j$ 's (and $a$ 's) bid(s) to the true valuation(s) here, and iteratively apply the above analysis to the other buyers in $C$ we have:

$$
\sum_{i \in C} u_{i}(\vec{b}) \leq \sum_{i \in C} u_{i}\left(\vec{b}^{\prime}\right)
$$

By the above analysis, we prove the lemma.

Lemma 7. Enhanced STAMP achieves individual-rationality and every buyer's payment is non-negative.

Proof: For a buyer $i$, assume that she bids truthfully by $b_{i}=v_{i}$. We divide the proof into two cases:

1) $i$ is allocated the item, then her utility is non-negative:

$$
\begin{aligned}
& u_{i}(\vec{b}) \\
= & v_{i} x_{i}-p_{i}(\vec{b}) \\
= & m(\vec{x}(\vec{b}, G), \vec{b})+\frac{k-1}{k} v_{i}-\hat{h} \\
\geq & \beta_{\text {inf }}+\frac{k-1}{k} v_{\text {inf }}-\hat{h} \\
\geq & 0 .
\end{aligned}
$$

Also, her payment is non-negative:

$$
\begin{aligned}
& p_{i}(\vec{b}) \\
= & \hat{h}-\frac{\sum_{j \in(\mathcal{W} \backslash\{i\})} b_{j}}{\hat{k}} \\
= & \hat{h}-m(\vec{x}(\vec{b}, G), \vec{b})+\frac{b_{i}}{\hat{k}} \\
\geq & \hat{h}-\beta_{\text {sup }}+\frac{v_{\text {inf }}}{\hat{k}} \\
\geq & 0 .
\end{aligned}
$$

2) $i$ is not allocated the item, then her utility is 0 :

$$
u_{i}(\vec{b})=0 .
$$

From the above analysis, we finish the proof.

Theorem 3. Enhanced STAMP achieves semi-group-strategyproofness.

Proof: This is clear from Lemma 5, Lemma 6 and the definition of semi-group-strategy-proof mechanism.

Notice that enhanced STAMP runs the same maximum independent set algorithm for initial item allocation. It achieves the same approximation ratio as STAMP in terms of allocation efficiency.

Theorem 4. Enhanced STAMP achieves an asymptotic approximation ratio of:

$$
\min \left\{\kappa / \mu,\left[\kappa^{\prime} \log (\log \Delta)\right] / \Delta\right\}
$$

(where $\kappa$ is a positive constant, $\kappa^{\prime}$ is a constant depends on $\kappa$, and $\Delta, \mu$ are the maximum and the average degrees of the $G$, respectively) in terms of allocation efficiency [6].

Proof: The proof is the same as Theorem 2.

It is a common case the seller has a set of heterogeneous spatially reusable items to sell (e.g., multiple tasks and spectrum) and a buyer have different valuations for each item. We can apply STAMP for the items separately to compute the allocation outcome. 


\section{Evaluation}

In this section, we implement STAMP and evaluate its performance in terms of allocation efficiency.

\section{A. Methodology}

We run the mechanism for over 1000 times to evaluate its performance. There are four different kinds of settings described later. In all these four settings, there is a single spatially reusable item for sale and the buyers' valuations lie in the range of $(0,1] .^{1}$ Here, we use a heuristic maximum independent set algorithm [2] in the initial allocation.

We investigate the allocation efficiency to measure the mechanism's performance. We compare the performances of STAMP with VERITAS and SMALL which are mechanisms for homogeneous spatially reusable items.

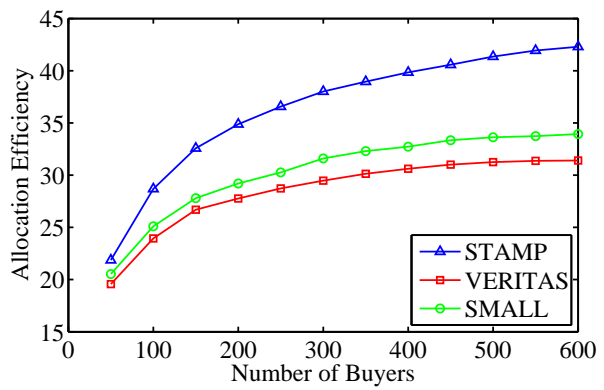

Fig. 2. Fixed Area and Varying Number of Buyers

First, we fix the terrain area to be 2000 meters $\times 2000$ meters, and the buyers are randomly distributed in this area. The number of buyers varies from 50 to 600 with step of 50. We also fix $d=300$ meters [4]. Figure 2 shows the comparison results on allocation efficiency of STAMP, VERITAS and SMALL. The results show that STAMP outperforms VERITAS and SMALL in terms of allocation efficiency in any situation. When the number of buyers increases, all mechanisms' allocation efficiency increase and the advantage of STAMP becomes more obvious, but the rate of increase drops since the average degree of $G$ also increases.

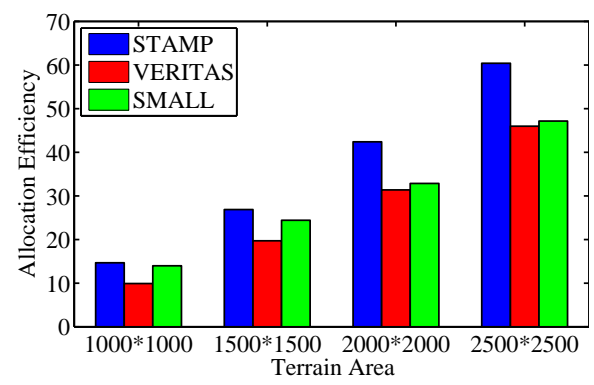

Fig. 3. Varying Area and Fixed Number of Buyers

\footnotetext{
${ }^{1}$ The ranges of buyers' valuations can be chosen differently from the ones used here. However, the evaluation results of using different ranges are similar to each other. As a result, we only show the results for the above ranges in this paper.
}

Second, we fix the number of buyers to be 500 and the terrain area varies from 1000 meters times 1000 meters to 2500 meters times 2500 meters with step of 1000 in side length and $d=300$ meters. Figure 3 shows the comparison results on allocation efficiency of STAMP, VERITAS and SMALL. The results show that STAMP outperforms VERITAS and SMALL in terms of allocation efficiency in the four terrain areas. When the area becomes larger, all mechanisms' allocation efficiency also increase and the differences between STAMP and VERTIAS, STAMP and SMALL all increase.

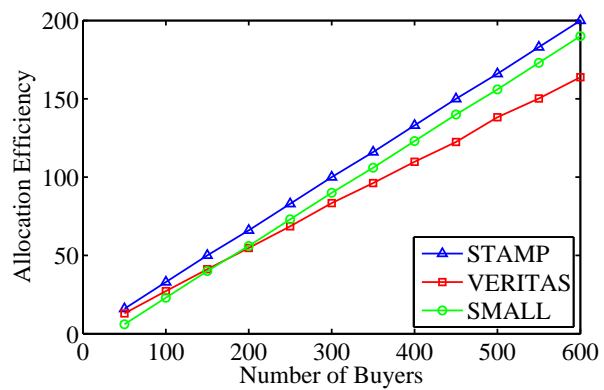

Fig. 4. Fixed Average Degree and Varying Number of Buyers

Third, we fix the average degree of the $G$ to be 4 , and the number of buyers varies from 50 to 600 with step of 50 . Figure 4 shows the comparison results on allocation efficiency of STAMP, VERITAS and SMALL for this setting. The results show that STAMP outperforms VERITAS and SMALL in terms of allocation efficiency. The difference of STAMP and VERTIAS increases along with the number of buyers while the difference of STAMP and SMALL remains the same all the time.

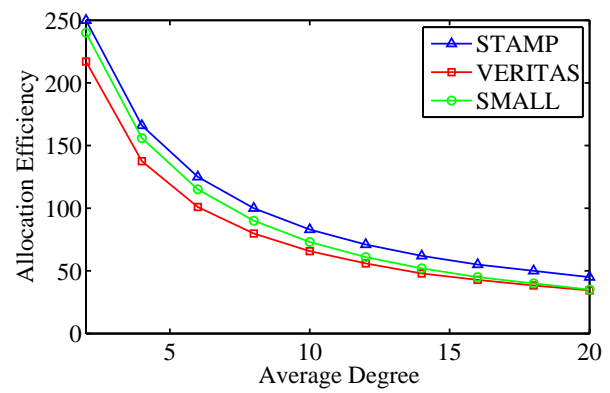

Fig. 5. Varying Average Degree and Fixed Number of Buyers

Finally, we fix the number of buyers to be 500 and the average degree of the $G$ varies from 2 to 20 with step of 2 . Figure 5 shows the comparison results on allocation efficiency of STAMP, VERITAS and SMALL for the setting. The results show that STAMP outperforms VERITAS and SMALL in terms of allocation efficiency when the average degree of $G$ varies. The allocation efficiency of all mechanisms decrease when the average degree of $G$ increases. This is because when the average degree of $G$ increases, the size of the maximum independent set of $G$ becomes smaller and so does the number of winners. 
From the above results, we can draw the conclusion that STAMP achieves superior performance in terms of allocation efficiency.

\section{CONCLUSION}

In this paper, we have introduced the problem of spatially reusable items allocation in which there was a single seller who wanted to sell spatially reusable items and performed an auction to allocate the item to a group of buyers. We have designed a strategy-proof approximation auction mechanism, namely STAMP, to solve the problem. STAMP achieves an allocation efficiency as high as the best ever known maximum independent set algorithm. We have further enhanced STAMP to achieve semi-group-strategy-proofness while preserving the same allocation efficiency as its previous version. For future work, we are going to consider auction mechanisms for some more challenging settings like online auction markets.

\section{REFERENCES}

[1] A. Archer and E. Tardos, "Truthful mechanisms for one-parameter agents," in FOCS, 2001.

[2] S. Balaji, V. Swaminathan, and K. Kannan, "A simple algorithm to optimize maximum independent set," AMO-Advanced Modeling and Optimization, vol. 12, no. 1, 2010.

[3] P. Berman and M. Frer, "Approximating maximum independent set in bounded degree graphs," in SODA, 1994.

[4] M. Carder, M. T. Thai, Y. Li, and W. Wu, "Energy-efficient target coverage in wireless sensor networks," in INFOCOM, 2005.

[5] G. Danezis, S. Lewis, and R. Anderson, "How much is location privacy worth?" in WEIS, 2005.

[6] M. Demange, "Improved approximations for maximum independent set via approximation chains," Applied Mathematics Letters, vol. 10, no. 3, pp. 105-110, 1997.

[7] D. Fudenberg and J. Tirole, Game Theory. MIT Press, 1991.

[8] A. Goldberg and J. Hartline, "Collusion-resistant mechanisms for singleparameter agents," in SODA, 2005.

[9] I. Hafalir, R. Ravi, and A. Sayedi, "Sort-cut: A pareto optimal and semi-truthful mechanism for multi-unit auctions with budget-constrained bidders." in $A A W, 2011$.

[10] R. Jurca and B. Faltings, "Collusion-resistant, incentive-compatible feedback payments," in ACM EC, 2007.

[11] A. Mas-Colell, M. D. Whinston, and J. R. Green, Microeconomic Theory. Oxford Press, 1995.

[12] J. McMillan, "Selling spectrum rights," Journal of Economic Perspectives, vol. 8, no. 3, pp. 145-162, 1994.

[13] M. J. Osborne and A. Rubenstein, A Course in Game Theory. MIT Press, 1994.

[14] P. Penna and C. Ventre, "Optimal collusion-resistant mechanisms with verification," in ACM EC, 2009.

[15] R. Rana, C. Chou, S. Kanhere, N. Bulusu, and W. Hu, "Earphone: An end-to-end participatory urban noise mapping," in ACM/IEEE IPSN, 2010.

[16] T. Sandholm, S. Suri, A. Gilpin, and D. Levine, "Cabob: A fast optimal algorithm for winner determination in combinatorial auctions," in IJCAI, 2001.

[17] J. Schummer, "Manipulation through bribes," Journal of Economic Theory, vol. 91, no. 3, pp. 180-198, 2000.

[18] Sensorly, http://www.sensorly.com.

[19] D. Wedelin, "An algorithm for large scale $0-1$ integer programming with application to airline crew scheduling," Annals of Operations Research, vol. 57, no. 1, pp. 283-301, 1995.

[20] F. Wu and N. Vaidya, "Small: A strategy-proof mechanism for radio spectrum allocation," in INFOCOM-mini, 2011.

[21] D. Yang, G. Xue, X. Fang, and J. Tang, "Crowdsourcing to smartphones: Incentive mechanism design for mobile phone sensing," in MobiCom, 2012.

[22] X. Zhou, S. Gandhi, S. Suri, and H. Zheng, "ebay in the sky: Strategyproof wireless spectrum auctions," in MobiCom, 2008. 